D. Henkel ${ }^{1}$ P. Grünbeck ${ }^{2}$

\section{Entwicklung der Arbeitslosenquote in der Suchtrehabilitation und Verlauf der beruflichen Integration Alkoholabhängiger vor und nach der Rehabilitation: Eine Auswertung von}

Development of the Unemployment Rate in Rehabilitation Programs and Course of Vocational Integration of Alcohol-addicted Patients before and after Rehabilitation: an Analysis of Routine Data of the Pension Insurances

\section{Zusammenfassung}

Fragestellung, Methode, Stichprobe: Anhand von Daten der gesetzlichen Rentenversicherungen wurde die Entwicklung des Anteils der Arbeitslosen in der Gruppe der alkoholabhängigen Rehabilitanden seit 1975 (VDR-Daten) untersucht und der Erwerbsverlauf im Zeitraum von 2 Jahren vor und 2 Jahren nach einer Suchtrehabilitation analysiert, unterteilt in 8 Messzeitpunkte (BfA-Daten, Reha-Jahrgang 2001, $n=5,929$ ). Ergebnisse: Es zeigte sich ein erheblicher Anstieg der Arbeitslosenquote von zunächst 7\% (1975) auf 37\% (2003). Dazu werden verschiedene Bedingungen diskutiert, die auf diese Entwicklung Einfluss genommen haben können. In Hinblick auf die berufliche Integration der Alkoholabhängigen fiel die Bilanz insofern negativ aus, als der Beschäftigtenanteil vor der Rehabilitation zwar kontinuierlich abgenommen hatte, aber zu Beginn des Untersuchungszeitraums höher war als nach der Reha-Maßnahme. Allerdings ließ sich feststellen, dass die Rehabilitation eine weitere Eskalation der beruflichen Desintegrationsprozesse verhindert hatte. Diese Verlaufsstruktur zeigte sich in West- und Ostdeutschland sowie bei Männern und Frauen gleichermaßen. Darüber hinaus wurde die Dauer der Arbeitslosigkeit und Erwerbstätigkeit nach der Rehabilitation in verschiedenen Gruppen untersucht (Männer-Frauen, Alters- und Einkommensgruppen u.a.m.). Die durchschnittliche Beschäftigungszeit variierte zwischen 12 und 18 Monaten. Die Mittelwerte der ununterbrochenen Arbeitslosigkeit lagen bei allen Arbeitslosengruppen dicht an der Grenze hin zur Langzeitarbeitslosigkeit oder hatten diese leicht überschritten. Schlussfolgerung: Die Ergebnisse unterstreichen, dass sowohl arbeitsreinte-

\section{Abstract}

Objective, method, sample: Based on data of the pension insurances this study investigates the development of the unemployment rate among alcohol-addicted patients in rehabilitation programs since 1975 (VDR-data) and analyses the course of vocational integration during a period of 2 years before and after treatment and rehabilitation, subdivided in 8 measurement points in time (BfA-data, rehabilitation cohort of the year 2001, $\mathrm{n}=5.929$ ). Results: It was proved to be a considerable increase of the unemployment rate from $7 \%$ (1975) to $37 \%$ in 2003 . Various conditions possibly having influenced this development are discussed. With regard to the vocational integration the outcome was negative in so far as the employment rate was much higher before than after rehabilitation. But it could be clearly established that rehabilitation prevented a further increase of vocational disintegration which had taken place during the period of 2 years before rehabilitation. The structure of this course of vocational integration among men and women and West and East Germany closely resembled each other. In addition to that the duration of employment and unemployment after rehabilitation was investigated for various groups (men-women, age and income groups etc.). The average length of employment varied between 12 and 18 months. The means of unemployment of all unemployed groups were near to the perimeter of long term unemployment or exceeded it slightly. Conclusion: The results underline that measures for promoting vocational reintegration and protecting against job loss must have a high priority in addiction treatment and rehabilitation.

${ }^{1}$ Fachhochschule Frankfurt a. M., Fachbereich 4 Soziale Arbeit und Gesundheit, Institut für Suchtforschung ISFF, University of Applied Sciences

${ }^{2}$ Deutsche Bundesversicherung Bund, Geschäftsbereich 0400 Sozialmedizin und Reha-Wissenschaften, Berlin 
grative Maßnahmen für die Arbeitslosen als auch arbeitsplatzsichernde für die Erwerbstätigen in der Suchtrehabilitation eine hohe Priorität haben müssen.

\section{Schlüisselwörter}

Alkoholabhängigkeit · gestiegene Arbeitslosenquote $\cdot$ berufliche Integration vor und nach Rehabilitation
Key words

Alcohol addiction - increased unemployment rate · vocational integration before and after rehabilitation

\section{Einleitung}

Maßnahmen der medizinischen Rehabilitation, so auch der Suchtrehabilitation, sind vor allem auf die Förderung der Teilhabe am Arbeitsleben gerichtet. Diese Zielsetzung ist besonders für Suchtkranke von Bedeutung, da der Erhalt der Erwerbstätigkeit bzw. eine berufliche Wiedereingliederung die Erreichung der suchtspezifischen Rehabilitationsziele in erheblichem Maße erleichtert, d.h. Sicherung der Abstinenz bzw. Minimierung von Rückfallrisiken. Wer nach der Suchtbehandlung für längere Zeit arbeitslos ist, hat im Vergleich zu den Erwerbstätigen ein rund 2fach höheres Risiko alkoholrückfällig zu werden und sogar ein 3,5fach höheres für eine Rückfälligkeit in die frühere Alkoholabhängigkeit [1 -2]. Unter dem Aspekt der Qualitätssicherung der Suchtrehabilitation (im Folgenden auch kurz: Reha) kommt daher der Frage eine herausragende Bedeutung zu, inwieweit es gelingt, Suchtbehandelte in die Arbeitswelt zu integrieren. Dies ist vor allem auch vor dem Hintergrund des Anteils der Arbeitslosen unter den Suchtrehabilitanden zu sehen, der, wie noch detailliert zu zeigen sein wird, im Zuge der Massenarbeitslosigkeit seit 1975 stark gestiegen ist und inzwischen mit über 35\% ein erhebliches Ausmaß erreicht hat.

\section{Bisherige Untersuchungen}

Bisherige Untersuchungen zur Entwicklung der Arbeitslosenquote erstreckten sich auf relativ kleine Zeiträume und Teilsegmente der Suchtrehabilitation, so z.B. die Erhebungen des Fachverbands Sucht, die erst mit dem Jahr 1996 begannen und an denen sich maximal 11 Fachkliniken für Suchtkranke beteiligten [3]. Die hier präsentierten Daten hingegen gehen bis in das Jahr 1975 zurück und umfassen den Gesamtbereich der medizinischen Suchtrehabilitation in der Trägerschaft der gesetzlichen Rentenversicherung.

Auch die bisherigen Studien zum Erwerbsverlauf bzw. beruflichen Integration nach der Suchtrehabilitation weisen Probleme bzw. Lücken auf. Die jährlich durchgeführten Katamnesestudien des Fachverbands Sucht [3] stützten sich zum einen nur auf subjektive, damit wahrscheinlich z.T. fehlerbehaftete Angaben zum Erwerbsstatus der Rehabilitanden und zum anderen lediglich auf die Katamneseteilnehmer, damit in der Regel nur auf Zweidrittel eines Entlassjahrgangs. Zudem wurde hinsichtlich des Erwerbsstatus bislang nicht zwischen verschiedenen Teilgruppen der Rehabilitanden differenziert, z. B. zwischen Männern und Frauen, Alters- und Einkommensgruppen.

Die Auswertungen von Daten der Bundesversicherungsanstalt für Angestellte (BfA) zum Erwerbsverlauf nach der Rehabilitation durch Hümmelink \& Grünbeck (2002) [4] hingegen basierten auf einer Vollerhebung eines Reha-Jahrgangs (1998, Pflichtversicherte, Alkoholabhängige) sowie auf einer Definition des Erwerbsstatus anhand der Beitragszahlungen zur Rentenversicherung, somit auf einem objektiven Kriterium. Als Ergebnis zeigte sich, dass während des Zeitraums 2 Jahre nach der Rehabilitation bei $62 \%$ der Rehabilitanden lückenlose und bei 26\% lückenhafte Beitragszahlungen vorlagen, $7 \%$ in Frührente wegen Berufs- oder Erwerbsunfähigkeit und 2\% in Altersrente gegangen und 3\% verstorben waren. Doch Beitragsleistungen allein bedeuten noch nicht eine tatsächlich erfolgte Reintegration in die Erwerbstätigkeit, da auch im Fall der Arbeitslosigkeit Beiträge entrichtet werden. Daher blieb in dieser Untersuchung noch offen, wie viele und wie lange tatsächlich eine Beschäftigung hatten bzw. arbeitslos waren.

Die vorliegende Studie untersucht den Erwerbsverlauf ebenfalls auf der Grundlage von rehabilitationsstatistischen Daten der BfA und differenziert dabei erstmals zwischen Beschäftigung und $\mathrm{Ar}$ beitslosigkeit.

\section{Fragestellungen, Datenbasis, Methodik}

Wenn nicht anders vermerkt, beziehen sich alle hier vorgestellten Daten auf die Gruppe der Alkoholabhängigen, die eine Maßnahme der medizinischen Suchtrehabilitation (Entwöhnungsbehandlung) in der Trägerschaft der gesetzlichen Rentenversicherungen absolvierten.

Die Ergebnisse zur Entwicklung der Arbeitslosenquote basieren auf einer Vollerhebung aller Rehabilitanden jeweils eines RehaJahrgangs, die mit der Diagnose Alkoholabhängigkeit (vor 2000 ICD-9 303, danach ICD-10 F10.2) in einer ambulanten oder stationären Maßnahme der Suchtrehabilitation waren. Die Daten umfassen sämtliche Versicherungszweige der gesetzlichen Rentenversicherung, die derzeit rund $85 \%$ aller Maßnahmen der medizinischen Suchtrehabilitation der Alkoholabhängigen in Deutschland tragen [3]. Sie stammen aus der vom Verband Deutscher Rentenversicherungsträger (VDR) jährlich erstellten VDRStatistik Rehabilitation [5]. Der hier untersuchte Status „arbeitslos“ bezieht sich auf den Zeitpunkt der Reha-Antragstellung. Die vorgestellte Datenreihe wird unter der Frage betrachtet: Welche Faktoren könnten auf den Anstieg der Arbeitslosenquote Einfluss genommen haben?

In Hinblick auf die berufliche (Re-)Integration vor und nach der Suchtrehabilitation werden 3 Fragestellungen untersucht:

1. Wie hatte sich der Erwerbsverlauf des Reha-Jahrgangs 2001, dem für die vorliegenden Untersuchungsziele aktuellsten statistisch verfügbaren Jahrgang, in den 2 Jahren vor und nach der Rehabilitation entwickelt, somit in der Zeitspanne von 
1999 bis 2003? Gibt es hier Unterschiede zwischen West- und Ostdeutschland sowie Männern und Frauen?

2. Wie sahen die Erwerbsverläufe im Zeitraum von 2 Jahren nach der Rehabilitation bei jenen beiden Gruppen aus, die vor allem aus der Sicht der Reha-Einrichtungen im Hinblick auf den Bedarf und die Effektivität arbeitsfördernder bzw. arbeitsplatzsichernder Maßnahmen im Mittelpunkt des Interesses stehen, nämlich diejenigen, die als Arbeitslose bzw. Erwerbstätige in die Behandlung kommen?

3. Wie lang war die durchschnittliche Arbeitslosigkeits- bzw. Beschäftigungsdauer im Zeitraum von 2 Jahren nach der Rehabilitation, differenziert nach Geschlecht, Region (West/ Ost), Alter, Einkommen, Arbeitslosigkeitsdauer und Arbeitsunfähigkeitszeiten vor Reha-Beginn.

Die empirische Grundlage für die Untersuchung dieser 3 Fragen bildet die so genannte Reha-Statistik-Datenbasis (RSD) der BfA, die jährlich routinemäßig erstellt wird [4]. Sie führt personenbezogene Daten aus den Bereichen Versicherung, Rehabilitation und Rente personenbezogen zusammen, umfasst eine Vielzahl soziodemografischer, krankheits- und erwerbsbezogener Merkmale (u.a. Geschlecht, Alter, Diagnose, Beiträge zur Rentenversicherung) und kann vor allem auch im Längsschnitt analysiert werden. Sie ermöglicht daher verlaufsorientierte Untersuchungen [6], so auch von Prozessen der beruflichen Integration im Zeitraum vor und nach der Suchtrehabilitation. In der vorliegenden Studie ist der Untersuchungszeitraum in 8 Messzeitpunkte untergliedert: 24, 12, 6 und 1 Monat(e) vor sowie 1, 6, 12 und 24 Monat(e) nach der Rehabilitation.

In der RSD wird der Erwerbsstatus in 3 Kategorien unterteilt: beschäftigt, arbeitslos und längerfristig arbeitsunfähig. Hinzu kommt das Merkmal: ausgeschieden aus dem Erwerbsleben durch Tod oder Rentenzugang. Die Zuordnung der Rehabilitanden zu einer der Statuskategorien erfolgt anhand der jeweiligen Art bzw. Herkunft der auf das Versicherungskonto pro Monat eingezahlten Rentenbeiträge bzw. durch Analyse der Rentendaten oder der Daten über Verstorbene. Danach waren die Rehabilitanden in dem Monat beschäftigt, in dem ein Arbeitgeber-/Arbeitnehmerbeitrag gezahlt wurde, und arbeitslos bei einer Einzahlung durch die Bundesagentur für Arbeit. Leistete eine Krankenkasse den Versicherungsbeitrag, so bestand eine so genannte längerfristige, d.h. über die tariflich festgelegte Zeit der Lohnfortzahlung im Krankheitsfall hinausgehende Arbeitsunfähigkeit. Die Arbeitsunfähigen sind aber in Wirklichkeit entweder arbeitslos oder beschäftigt. Dies ist jedoch in der RSD nicht erkennbar. Daher besteht hier eine gewisse Unsicherheit in der Zuordnung der Arbeitsunfähigen zu den Arbeitslosen und Erwerbstätigen, wenngleich aufgrund weiterer Datenquellen der BfA davon auszugehen ist, dass sich die längerfristig Arbeitsunfähigen etwa je zur Hälfte aus Arbeitslosen und Beschäftigten zusammensetzten (nicht veröffentlichte Analysen der BfA, Dezernat 8012).

Die aus der RSD für die vorliegende Untersuchung gezogene Stichprobe umfasst alle Rehabilitanden mit der Diagnose Alkoholabhängigkeit (ICD-10 F10.2), die im Jahr 2001 aus einer stationären Suchtrehabilitation entlassen wurden und den Status „Pflichtversicherte“ hatten, d.h. im Jahr vor der Reha-Antragstellung mindestens einen Monatsbeitrag zur Rentenversicherung leisteten. Insgesamt handelt es sich um 5929 Personen, darunter
3485 Männer und 2444 Frauen mit einem Durchschnittsalter von rund 44 bzw. 43 Jahren.

\section{Ergebnisse}

Zunächst werden die Ergebnisse zur Entwicklung der Arbeitslosenquote in der Suchtrehabilitation von 1975 bis 2003 dargestellt. Abb. 1 zeigt, dass der Anteil der Arbeitslosen in der Gruppe der Alkoholabhängigen von rund 7\% im Jahr 1975 kontinuierlich auf 37\% im Jahr 2003 gestiegen ist, wobei die mit Abstand stärkste Zunahme in der ersten Hälfte der 1980er-Jahre stattfand. Zudem fällt auf, dass die Quote während des gesamten Zeitraums weit über der allgemeinen Arbeitslosenquote in Deutschland lag, von 1975 bis 1990 sogar zunehmend stärker als diese angestiegen war und sich danach auf einem überproportional hohen Niveau von rund 35-37\% eingependelt hat. Diese Entwicklung kann verschiedene Ursachen haben:

1. Wenngleich für Deutschland keine repräsentativen empirischen Fakten vorliegen, so ist doch davon auszugehen, dass Alkoholabhängige in Zeiten hoher Arbeitslosigkeit und damit verschärfter Personalauslese in den Betrieben und Verwaltungen überdurchschnittlich häufig arbeitslos werden infolge hoher sucht- bzw. krankheitsbedingter Fehlzeiten, Leistungsminderungen, disziplinarischer Konflikte u.a.m., so dass die Quote der alkoholabhängigen Arbeitslosen stärker angewachsen ist als die allgemeine Arbeitslosenquote.

2. Zudem sind die Arbeitslosenquoten in jenen Gruppen seit Beginn der 1980er-Jahre überdurchschnittlich gestiegen, die zu den so genannten Problemgruppen des Arbeitsmarkts gehören [8] und aus denen sich alkoholabhängige Arbeitslose in der Suchtrehabilitation relativ häufig rekrutieren: Ältere, beruflich gering Qualifizierte und gesundheitlich Eingeschränkte $[3,9]$.

3. Auch Kumulationseffekte durch Wiederholungen von Suchtrehabilitationsmaßnahmen spielen eine wesentliche Rolle. Denn arbeitslose Alkoholabhängige nehmen Suchtbehand-

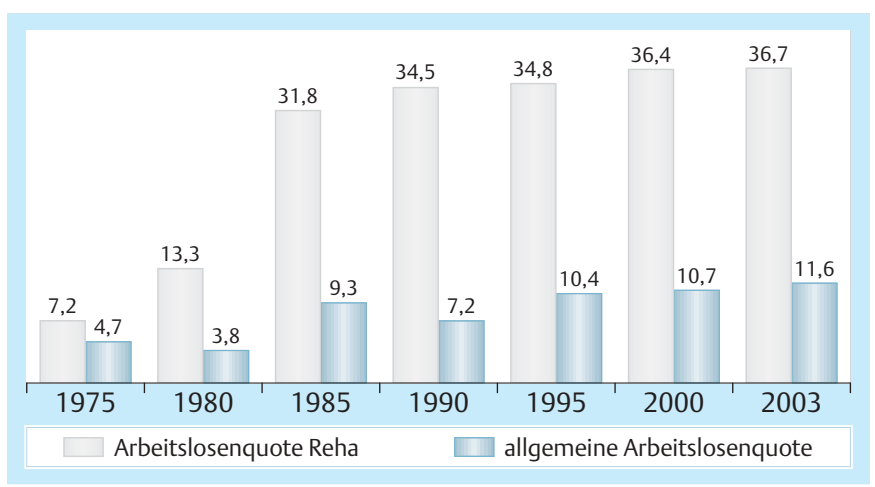

Abb. 1 Arbeitslosenquoten der Alkoholabhängigen in der Suchtrehabilitation in der Trägerschaft der gesetzlichen Rentenversicherung und allgemeine Arbeitslosenquote in Deutschland von 1975 bis 2003, bis 1990 früheres Bundesgebiet, ab 1995 Gesamtdeutschland. Anmerkung: Alkoholabhängigkeit vor 2000 nach ICD-9 303, ab 2000 nach ICD-10 F10.2; Status „arbeitslos“ bei Reha-Antragstellung; Arbeitslosenquote Reha: Arbeitslose in \% aller Rehabilitanden, allgemeine Arbeitslosenquote: Arbeitslose in \% der Erwerbspersonen (Erwerbstätige plus Arbeitslose), Quelle: VDR-Statistik Rehabilitation [5]; Bundesministerium für Arbeit und Sozialordnung [6]. 
lungen infolge der erheblich höheren Rückfallquote häufig wiederholt in Anspruch [2]. Bezöge man die Arbeitslosenquote nur auf Erstbehandelte, so läge sie wesentlich niedriger, in den letzten 10 Jahren mindestens um ein Drittel [2].

4. Wahrscheinlich war auch von Einfluss, dass der Anteil der Langzeitarbeitslosen seit Anfang der 1980er-Jahre stark gestiegen ist [8] und infolgedessen vermutlich auch der Anteil der behandlungsbedürftigen Alkoholabhängigen in der Gruppe der Arbeitslosen. Denn Längsschnittstudien weisen nach, dass ausgeprägte Alkoholprobleme, die bereits vor Beginn der Arbeitslosigkeit bestanden, sich im Zuge lang anhaltender Arbeitslosigkeit signifikant häufiger intensivieren als während eines gleich langen Zeitraums kontinuierlicher Beschäftigung. Dieser Zusammenhang ist zumindest für Männer nachgewiesen [10-11].

5. Möglicherweise ist die Zunahme der Arbeitslosenquote in der Suchtrehabilitation auch auf verstärkte Interventionen der Arbeitsagenturen zurückzuführen. Denn liegt eine Suchtproblematik vor, kann die Arbeitslosenunterstützung eingeschränkt oder entzogen werden, wenn keine Behandlung aufgenommen wird. Immerhin wurde im Jahr 2001 in rund 27500 $(8,9 \%)$ der insgesamt 310000 arbeitsamtsärztlichen Gutachten als Erstdiagnose eine „psychische und Verhaltensstörung durch psychotrope Substanzen“ (ICD-10 F1) festgestellt [12]. Allerdings fehlen Vergleichszahlen aus früheren Jahren.

Hohe Arbeitslosenquoten in der Suchtrehabilitation lassen sich sowohl bei Männern und Frauen als auch bei anderen Suchtdiagnosegruppen feststellen (Abb. 2). In Hinblick auf die absoluten Fallzahlen jedoch bildeten im Jahr 2003 die arbeitslosen Alkoholabhängigen mit 14045 von insgesamt 38259 alkoholabhängigen Rehabilitanden die mit Abstand größte Gruppe. Zum Vergleich: arbeitslose Drogenabhängige 3245 (alle 8662), Medikamentenabhängige 111 (alle 425), Mehrfachabhängige 1462 (alle 3777) [5].

Eine überproportional starke Inanspruchnahme suchtspezifischer Gesundheitsleistungen durch Arbeitslose zeigt sich auch bei Behandlungsmaßnahmen in der Trägerschaft der Krankenversiche- rungen. Grobe \& Schwartz (2003) [13] werteten Daten der Gmünder Ersatzkasse mit bundesweit 1,3 Millionen Versicherten aus dem Jahr 2000 aus und fanden, dass rund 14\% aller Krankenhaustage der arbeitslosen Männer (Frauen 2,5\%) allein auf die ICD10-Diagnose „psychische und Verhaltensstörungen durch Alkohol" entfielen. Die unter dieser Diagnose verbrachte Zahl der Krankenhaustage der arbeitslosen Männer lag um das 10fache (Frauen 5fache) höher als bei den Erwerbstätigen.

\section{Ergebnisse zur beruflichen Integration vor und nach der Rehabilitation}

\section{Zur Frage 1}

Abb. 3 bezieht sich auf Westdeutschland und zeigt die Verteilung des Erwerbsstatus der Rehabilitanden zu 8 Zeitpunkten vor und nach der Reha-Maßnahme. Nimmt man die längerfristig Arbeitsunfähigen zunächst aus der Betrachtung heraus, so ist beim Vergleich der beiden Zeiträume 2 Jahre vor und 2 Jahre nach der

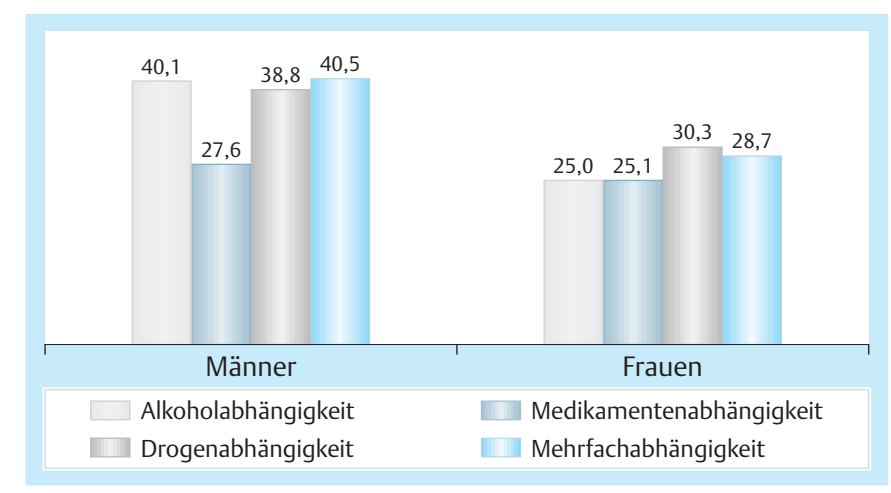

Abb. 2 Arbeitslosenquoten in der Suchtrehabilitation in der Trägerschaft der gesetzlichen Rentenversicherung nach Geschlecht und Diagnosegruppen im Jahr 2003. Anmerkung: Status „arbeitslos“ bei RehaAntragstellung, Arbeitslosenquote Reha: Arbeitslose in \% aller Rehabilitanden, allgemeine Arbeitslosenquote: Arbeitslose in \% der Erwerbspersonen (Erwerbstätige plus Arbeitslose), Diagnosen nach ICD-10, Quelle: VDR-Statistik Rehabilitation [5].

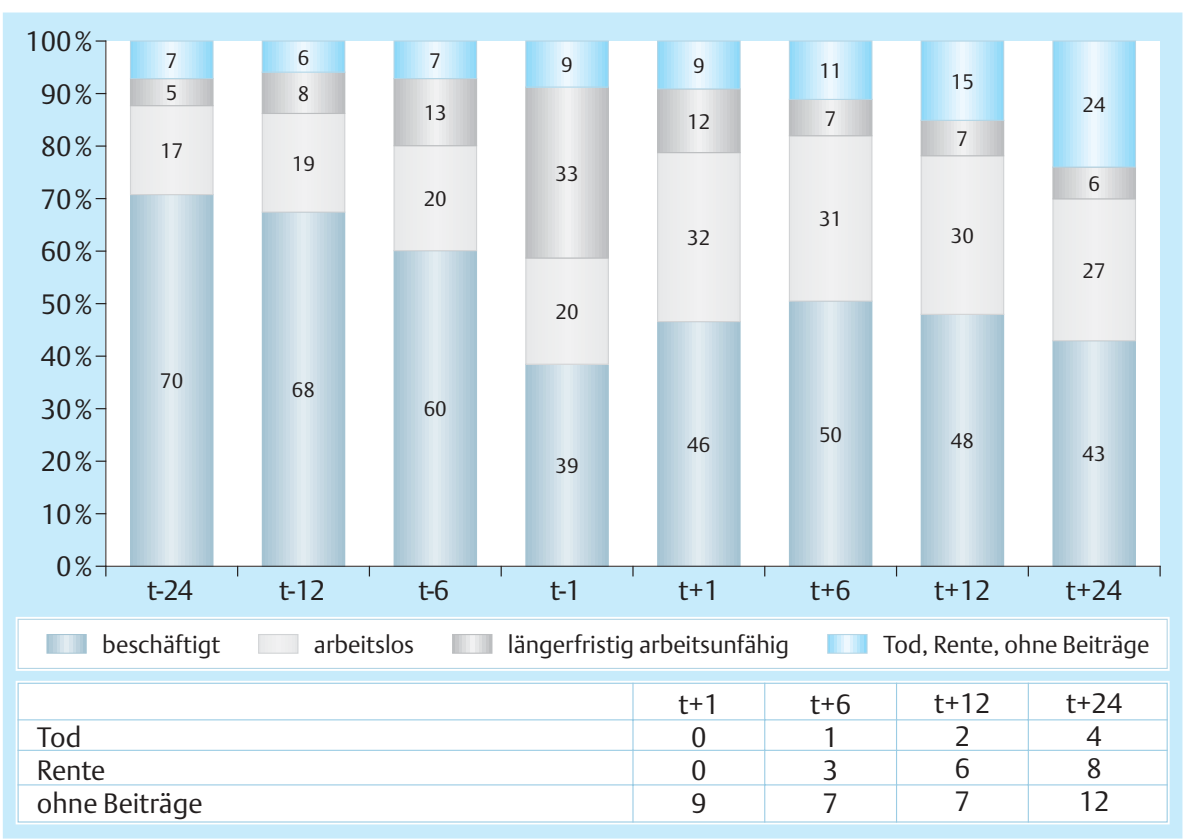

Abb. 3 Prozentuale Verteilung des Erwerbstatus im Zeitraum von 2 Jahren vor und nach der Suchtrehabilitation, Alkoholabhängige ICD-10 F10.2, BfA-Reha-Jahrgang 2001 Westdeutschland $(n=4,972)$, t- Monate vor Reha, $\mathrm{t}+$ Monate nach Reha. Quelle: RSD der BfA 1996-2003 
Rehabilitation zu erkennen, dass die berufliche Situation der Rehabilitanden vorher deutlich besser war. Bezogen auf den Anfangs- und Endzeitpunkt ( $t-24, t+24)$ ergibt sich per saldo ein Zuwachs des Arbeitslosenanteils um 10 und eine Abnahme der Beschäftigtenquote um 27 Prozentpunkte.

Unter der begründeten Annahme, dass die Hälfte der längerfristig Arbeitsunfähigen arbeitslos war, würde sich in Westdeutschland ein wesentlich stärkerer Anstieg der Arbeitslosenquote vor RehaBeginn von 19,5 auf 36,5\% zeigen und danach eine Abnahme von 38 auf 30\%: Gesamtveränderung per saldo plus 10,5 Prozentpunkte. Hinsichtlich des Beschäftigtenanteils ergäbe sich ein kontinuierlicher Rückgang von anfänglich 72,5 auf 55,5\% und nach RehaEnde eine weitere, aber vergleichsweise moderate Abnahme von $52 \%$ auf schließlich $46 \%$ : Gesamtveränderung per saldo minus 26,5 Prozentpunkte. Besonders augenscheinlich werden diese Verlaufsstrukturen in Abb.4, die ohne die Gruppe „berentet, verstorben oder ohne Beiträge“ berechnet sind.
Betrachtet man die längerfristig Arbeitsunfähigen als eigenständige Gruppe (Abb. 3), so nahm deren Anteil hin zum Beginn der Reha-Maßnahme stetig zu und danach etwa im gleichen Umfang wieder ab. Demgegenüber stieg der Anteil der Rehabilitanden, die im jeweiligen Untersuchungsmonat berentet oder (nach Reha) verstorben oder ohne Beiträge waren, kontinuierlich von $7 \%$ auf schließlich $24 \%$, wobei der stärkste Zuwachs in der Gruppe der Berenteten (Früh- und Altersrenten) zu verzeichnen war (Abb.3). Der Anteil der Frühberentungen wegen Erwerbs- oder Berufsunfähigkeit betrug 2 Jahre nach der Rehabilitation 6,4\% (Männer 6,3\%, Frauen $6,5 \%$.

Ein ähnlicher Erwerbsverlauf wie in Westdeutschland war auch in Ostdeutschland festzustellen, aber erwartungsgemäß mit durchgängig höheren Arbeitslosenquoten (Abb. 5 und 6). Auch der Anteil der Frühberenteten war mit 8,7\%2 Jahre nach der Rehabilitation etwas höher (Männer 9,1\%, Frauen 8,0\%).
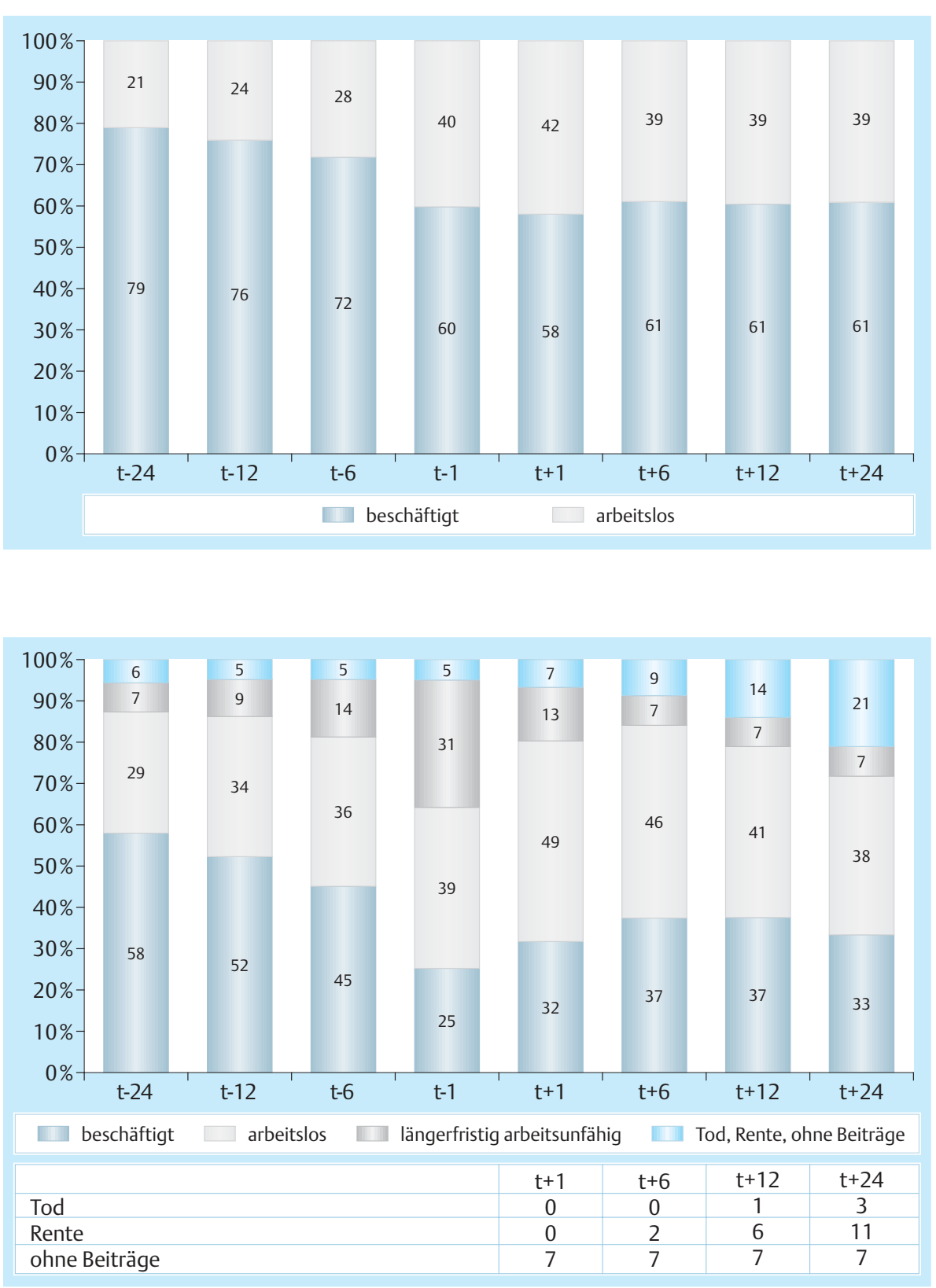

Abb. 4 Prozentuale Verteilung des Beschäftigten- und Arbeitslosenanteils bei je hälftiger Zuordnung der längerfristig Arbeitsunfähigen im Zeitraum von 2 Jahren vor und nach der Suchtrehabilitation, Alkoholabhängige ICD-10 F10.2, BfA-Reha-Jahrgang 2001 Westdeutschland $(n=4,972)$, tMonate vor Reha, $\mathrm{t}+$ Monate nach Reha. Anmerkung: ohne die Gruppe „berentet, verstorben oder ohne Beiträge“, Quelle: RSD der BfA 1996-2003

Abb. 5 Prozentuale Verteilung des Erwerbsstatus im Zeitraum von 2 Jahren vor und nach der Suchtrehabilitation, Alkoholabhängige ICD-10 F10.2, BfA-Reha-Jahrgang 2001 Ostdeutschland $(\mathrm{n}=957)$, t- Monate vor Reha, t+ Monate nach Reha. Quelle: RSD der BfA 1996-2003 

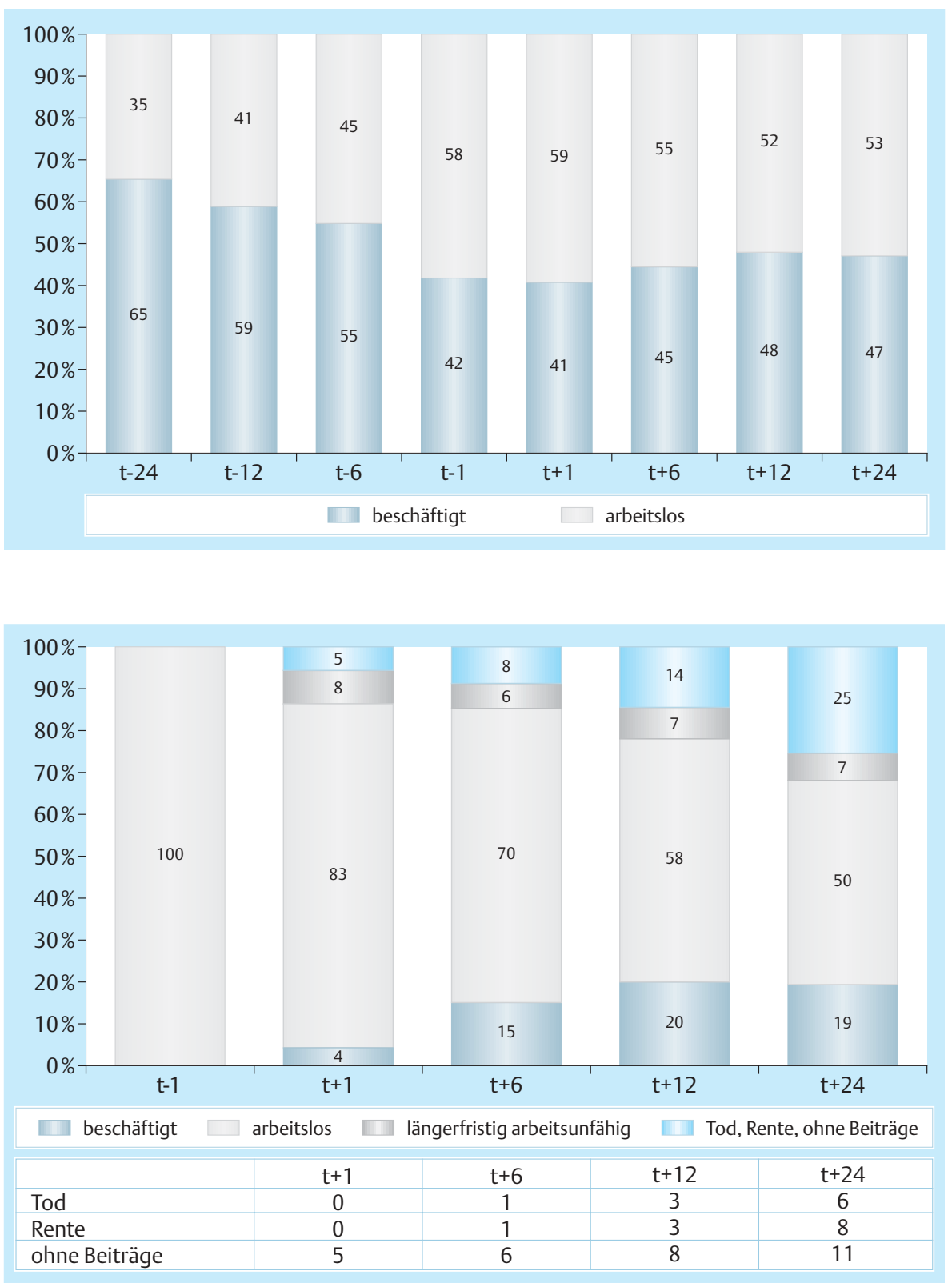

Abb. 6 Prozentuale Verteilung des Beschäftigten- und Arbeitslosenanteils bei je hälftiger Zuordnung der längerfristig Arbeitsunfähigen im Zeitraum von 2 Jahren vor und nach der Suchtrehabilitation, Alkoholabhängige ICD-10 F10.2, BfA-Reha-Jahrgang 2001 Ostdeutschland ( $n=957)$, $t-$ Monate vor Reha, t+ Monate nach Reha. Anmerkung: ohne die Gruppe „berentet, verstorben oder ohne Beiträge“, Quelle: RSD der BfA 1996-2003
Abb. 7 Prozentuale Verteilung des Erwerbsstatus im Zeitraum von 2 Jahren nach der Suchtrehabilitation, Alkoholabhängige ICD-10 F10.2, BfA-Reha-Jahrgang 2001, Teilgruppe „1 Monat vor Reha arbeitslos“ $(n=1,342)$, $t-$ Monat vor Reha, $t+$ Monate nach Reha. Quelle: RSD der BfA 1996-2003
Zwischen Männern und Frauen zeigten sich keine nennenswerten Unterschiede, weder in der Erwerbsverlaufsstruktur noch in der Höhe der Arbeitslosen- und Beschäftigtenquoten (ohne Abb.). Das gilt für West- und Ostdeutschland gleichermaßen.

\section{Zur Frage 2}

Nachfolgend werden aus dem Reha-Jahrgang 2001 jene Teilgruppen betrachtet, die im ersten Monat vor Reha-Beginn arbeitslos bzw. beschäftigt waren, also jene alkoholabhängigen Rehabilitanden, die als Arbeitslose bzw. Erwerbstätige in die Suchtbehandlung kamen.

Abb. 7 zeigt für die Gruppe der zu t-1 Arbeitslosen insofern einen positiven Verlauf, als deren Arbeitslosenquote sich bereits im ersten Monat nach Reha um 17 Prozentpunkte verringerte und danach weiter und kontinuierlich auf schließlich $50 \%$ sank. Allerdings stieg der Anteil derer, die wieder einen Arbeitsplatz hatten, nicht spiegelbildlich an, sondern von zunächst $4 \%$ auf schließlich nur $19 \%$. Zusätzlich ist zu sehen, dass am Ende des Unter- suchungszeitraums ein Viertel aus dem Erwerbsleben ausgeschieden waren, d.h. berentet oder verstorben oder ohne Beiträge waren.

Bei den zu t-1 Erwerbstätigen hingegen war der Verlauf durchgehend negativ (Abb. 8). Der Beschäftigtenanteil nahm schon im ersten Monat nach Reha um 16 Prozentpunkte ab und danach stetig auf schließlich $67 \%$. Gleichzeitig verdoppelte sich die Arbeitslosenquote von 7 auf $15 \%$.

Der Anteil der Arbeitsunfähigen war in beiden Erwerbsstatusgruppen annähernd gleich hoch und hatte sich über die Zeit nicht wesentlich verändert (Abb. $\mathbf{7}$ und $\mathbf{8}$ ).

\section{Zur Frage 3}

Tab. 1 zeigt die durchschnittliche Dauer der ununterbrochenen Beschäftigung, Arbeitslosigkeit und Arbeitsunfähigkeit im Zeitraum von 2 Jahren nach der Suchtrehabilitation, differenziert nach verschiedenen Gruppen. Getrennt nach den 3 Erwerbsstatusgruppen 


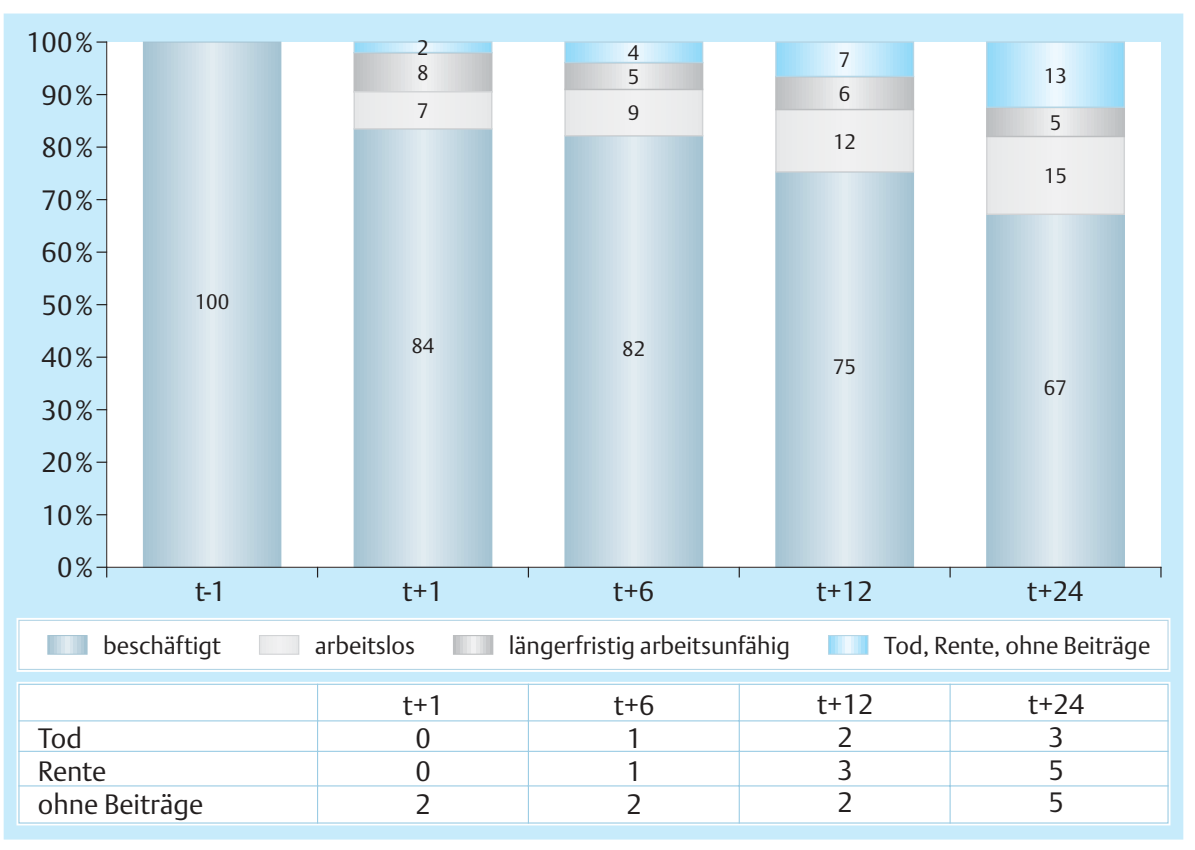

Abb. 8 Prozentuale Verteilung des Erwerbsstatus im Zeitraum von 2 Jahren nach der Suchtrehabilitation, Alkoholabhängige ICD-10 F10.2, BfA-Reha-Jahrgang 2001, Teilgruppe „1 Monat vor Reha beschäftigt“ $(n=2,199)$, $t-$ Monat vor Reha, $t+$ Monate nach Reha. Quelle: RSD der BfA 1999-2003

im ersten Monat nach Reha-Ende lassen sich die Ergebnisse wie folgt zusammenfassen:

Die zu t+ 1 Beschäftigten blieben durchschnittlich mindestens 12 Monate lang erwerbstätig; oft liegt der Mittelwert sogar über 15 Monaten und mehr. Die beste Bilanz zeigt sich bei der oberen Einkommensgruppe und den vor Reha-Beginn unter 3 Monaten Arbeitsunfähigen, die schlechteste bei denen, die im Jahr vor RehaBeginn länger als 183 Tage (6 Monate) arbeitslos waren. Innerhalb der einzelnen Gruppen bestanden statistisch bedeutsame Differenzen. Die Frauen, die untere Einkommensgruppe und die vor Reha-Beginn über 6 Monate lang Arbeitunfähigen hatten in Relation zu den jeweiligen Vergleichsgruppen signifikant kürzere Beschäftigungszeiten.

Die verschiedenen Gruppen der zu t+ 1 Arbeitslosen blieben im Mittel rund 12 bis 14 Monate lang ununterbrochen ohne Arbeit. Die schlechtesten Resultate hatten die Rehabilitanden in Ostdeutschland, die über 50-Jährigen und diejenigen mit einer $\mathrm{Ar}$ beitslosigkeit von 6 bis 12 Monaten im Jahr vor der Rehabilitation. Innerhalb der Gruppen zeigten sich signifikant längere Arbeitslosigkeitszeiten bei den Älteren und den Rehabilitanden in Ostdeutschland.

Hingegen sind in der Gruppe derer, die im ersten Monat nach Reha-Ende zu den längerfristig Arbeitsunfähigen gehörten, keinerlei nennenswerte Differenzen hinsichtlich der ununterbrochenen $\mathrm{Ar}$ beitsunfähigkeitsdauer nach der Rehabilitation zu erkennen, weder zwischen noch innerhalb der Gruppen. Sie betrug im Mittel rund 4 bis $4 \frac{1}{2}$ Monate.

\section{Diskussion}

Die präsentierten Ergebnisse belegen einen erheblichen Anstieg der Arbeitslosenquote in der Suchtrehabilitation auf inzwischen über 35\%. Vor dem Hintergrund derzeitiger Arbeitsmarktprognosen, die bis zum Jahr 2015 nur einen langsamen und insgesamt lediglich moderaten Zuwachs in der Arbeitskräftenachfrage und auch nur für Westdeutschland vorhersagen [14-15], ist in absehbarer Zeit eine deutliche Abnahme der Arbeitslosenquote auch im Bereich der Suchtrehabilitation eher unwahrscheinlich. Daher haben auf Suchtkranke zugeschnittene Maßnahmen zur Förderung der Teilhabe am Arbeitsleben noch für lange Zeit eine hohe Priorität, vor allem in Ostdeutschland. Letzteres unterstreichen auch die hier vorgestellten Ergebnisse, die auf deutlich schlechtere berufliche Integrationschancen im Osten Deutschlands verweisen.

Die eingangs dargestellten Ergebnisse der Untersuchung von Hümmelink \& Grünbeck (2002) [4] für den Reha-Jahrgang 1998 belegten, dass ein wesentliches Ziel der Suchtrehabilitation, nämlich die Verhinderung des vorzeitigen Ausscheidens aus dem Erwerbsleben durch Erwerbs- oder Berufsunfähigkeit, in hohem Maße erreicht wird, da der Anteil der Frühberentungen lediglich bei $7 \%$ lag. Auch in der vorliegenden Untersuchung des Reha-Jahrgangs 2001 betrug er insgesamt nur 6,7\%. Diese Bilanz ist vor allem dann eindeutig positiv, wenn man dabei von der plausiblen Annahme ausgeht, dass ein Rehabilitand definitionsgemäß bei Reha-Antragstellung von Erwerbsunfähigkeit bedroht war und bei Nicht-Durchführung einer Rehabilitation über kurz oder lang aus dem Erwerbsleben ausgeschieden und in Frühberentung gegangen wäre [4].

Misst man jedoch wie in der vorliegenden Studie die Erfolgsbilanz am Kriterium der tatsächlichen Integration in Beschäftigung, so ergibt sich eine andere Bilanz:

1. Sie erscheint zunächst eindeutig negativ, wenn man die Persaldo-Differenzen der Arbeitslosen- und Beschäftigtenanteile vor und nach der Suchtrehabilitation betrachtet und dabei die längerfristig Arbeitsunfähigen als eigenständige Gruppe berücksichtigt (Abb. 3,5).

2. Bei einem differenzierteren Blick auf den Erwerbsverlauf zeigt sich jedoch: Im Gegensatz zum Zeitraum vor der Rehabilitation sind danach keine weiteren stetigen Abnahmen der Beschäftigten- und Zunahmen der Arbeitslosenanteile festzustellen. Dieses positive Resultat, das sich in Ost- und 
Tab. 1 Dauer der ununterbrochenen Beschäftigung (B), Arbeitslosigkeit (A) und Arbeitsunfähigkeit (U) im Zeitraum von 2 Jahren nach der Suchtrehabilitation beginnend mit dem ersten Monat nach Reha ( $t+1)$, Alkoholabhängige ICD-10 F10.2, BfA-Reha-Jahrgang 2001, differenziert nach verschiedenen Gruppen $(n=5,574)$

\begin{tabular}{|c|c|c|c|c|c|c|c|}
\hline \multirow[t]{2}{*}{ Gruppen $^{1}$} & \multirow{2}{*}{$\begin{array}{l}\text { Erwerbsstatus } \\
\text { in } t^{+} 1\end{array}$} & \multirow{2}{*}{$\begin{array}{l}n \\
\text { in } t+1\end{array}$} & \multirow{2}{*}{$\begin{array}{l}\text { Anteil } \\
\text { in } t+1 \\
\%\end{array}$} & \multicolumn{2}{|c|}{ Dauer der $B, A, U$ in Monaten } & \multirow[t]{2}{*}{$t$} & \multirow[t]{2}{*}{$p$} \\
\hline & & & & $M$ & $S D$ & & \\
\hline \multicolumn{8}{|l|}{ Geschlecht } \\
\hline Männer & beschäftigt & 1607 & 48 & B 17,8 & 8,3 & 4,176 & $<0,01$ \\
\hline Frauen & & 1068 & 48 & B 16,4 & 8,9 & & \\
\hline Männer & arbeitslos & 1330 & 40 & A 12,7 & 8,5 & 2,569 & ns \\
\hline Frauen & & 820 & 37 & A 11,7 & 8,5 & & \\
\hline Männer & längerfristig arbeitsunfähig & 422 & 13 & U 4,3 & 4,0 & 0,067 & ns \\
\hline Frauen & & 327 & 15 & U 4,3 & 3,8 & & \\
\hline \multicolumn{8}{|c|}{ Ost-/Westdeutschland } \\
\hline Ost & beschäftigt & 309 & 34 & B 16,5 & 9,0 & 1,555 & ns \\
\hline West & & 2366 & 51 & B 17,3 & 8,5 & & \\
\hline Ost & arbeitslos & 483 & 53 & A 13,6 & 8,5 & 3,829 & $<0,01$ \\
\hline West & & 1667 & 36 & A 11,9 & 8,5 & & \\
\hline Ost & längerfristig arbeitsunfähig & 115 & 13 & U 4,2 & 4,0 & 0,272 & ns \\
\hline West & & 634 & 14 & U 4,3 & 3,9 & & \\
\hline $\begin{array}{l}\text { Alter in Jahres } \\
\text { unter } 50\end{array}$ & beschäftigt & 1772 & 46 & В 17,1 & 8,7 & 0,917 & ns \\
\hline über 50 & Destriartye & 903 & 53 & B 17,5 & 8,3 & & \\
\hline unter 50 & arbeitslos & 1609 & 42 & A 11,9 & 8,5 & 4,094 & $<0,01$ \\
\hline über 50 & & 541 & 31 & A 13,6 & 8,6 & & \\
\hline unter 50 & längerfristig arbeitsunfähig & 473 & 12 & U 4,1 & 4,0 & 1,653 & ns \\
\hline über 50 & & 276 & 16 & U 4,6 & 3,8 & & \\
\hline \multicolumn{8}{|l|}{ Einkommen $^{2}$} \\
\hline unter $50 \%$ & beschäftigt & 743 & 42 & B 15,1 & 9,2 & 8,517 & $<0,01$ \\
\hline über $50 \%$ & & 1888 & 68 & B 18,2 & 8,1 & & \\
\hline unter $50 \%$ & arbeitslos & 789 & 44 & A 10,9 & 8,5 & 1,348 & ns \\
\hline über $50 \%$ & & 518 & 19 & A 11,6 & 8,2 & & \\
\hline unter $50 \%$ & längerfristig arbeitsunfähig & 255 & 14 & U 4,3 & 3,6 & 0,066 & ns \\
\hline über $50 \%$ & & 372 & 13 & U 4,3 & 3,9 & & \\
\hline \multicolumn{8}{|c|}{ Arbeitslosigkeitszeiten im Jahr vor Reha in Tagen } \\
\hline unter 183 & beschäftigt & 2608 & 59 & B 17,4 & 8,5 & 4,676 & $<0,01$ \\
\hline über 183 & & 67 & 6 & B 12,4 & 9,1 & & \\
\hline unter 183 & arbeitslos & 1193 & 27 & A 11,0 & 8,3 & 8,158 & $<0,01$ \\
\hline über 183 & & 957 & 82 & A 14,0 & 8,5 & & \\
\hline unter 183 & längerfristig arbeitsunfähig & 611 & 14 & U 4,3 & 4,0 & 0,242 & ns \\
\hline über 183 & & 138 & 12 & U 4,4 & 3,5 & & \\
\hline \multicolumn{8}{|c|}{ Arbeitsunfähigkeitszeiten vor Reha in Monaten } \\
\hline unter 3 & beschäftigt & 1935 & 57 & B 18,1 & 8,2 & 5,664 & $<0,01$ \\
\hline 6 und mehr & & 192 & 27 & B 14,5 & 9,3 & & \\
\hline unter 3 & arbeitslos & 1132 & 34 & A 12,7 & 8,7 & 0,724 & ns \\
\hline 6 und mehr & & 303 & 42 & A 12,3 & 8,0 & & \\
\hline unter 3 & längerfristig arbeitsunfähig & 300 & 9 & U 4,5 & 4,2 & 1,311 & ns \\
\hline 6 und mehr & & 229 & 32 & U 4,0 & 3,2 & & \\
\hline
\end{tabular}

1 ohne die Gruppe „berentet, verstorben oder ohne Beiträge“

2 hochgerechnetes Einkommen im Jahr vor Reha-Antrag in \% der Beitragsbemessungsgrenze, M Mittelwert, SD Standardabweichung, t-Test, Signifikanzniveau < 0,05, ns nicht signifikant, Quelle: RSD der BfA 1996-2003

Westdeutschland und ebenso bei Männern und Frauen zeigt und als Effekt der Rehabilitation interpretiert werden kann, ist allerdings erst dann hinreichend klar ausgeprägt, wenn man die längerfristig Arbeitsunfähigen je zur Hälfte den $\mathrm{Ar}$ beitslosen und Beschäftigten zuordnet (Abb. 4,6). Dann dokumentieren die Ergebnisse, dass der Effekt der Suchtrehabilitation darin bestand, dass sie eine weitere Eskalation der beruflichen Desintegrationsprozesse verhinderte. Allerdings blieben die Arbeitslosenquoten mehr oder weniger konstant hoch und die Beschäftigtenanteile niedrig, so dass von einer Trendumkehr keine Rede sein kann.

Dabei muss man allerdings in Rechnung stellen, dass in der Zeit von 2001 bis 2003, also in dem hier betrachteten Zeitraum während und nach der Rehabilitation, sich einige Eckwerte des Arbeitsmarkts deutlich verschlechtert hatten. So ist vor allem festzustellen: ein Anstieg der Arbeitslosenquo- 
te um 1,3 Prozentpunkte (West 1,3, Ost 1,3). Das bedeutete eine Zunahme der registrierten Arbeitslosen um 524000 (West 432 000, Ost 92000 ) [14], wobei die Zahl der Langzeitarbeitslosen überproportional anwuchs und sich in Ostdeutschland sogar nahezu verdoppelte [14]. Hinzu kam ein starker Abbau der Arbeitsbeschaffungsmaßnahmen in Ost und West, deren Anzahl im Osten um fast 50\% zurückging [16].

3. In der Gruppe derjenigen, die die Suchtbehandlung als Arbeitslose begannen, wies der Erwerbsverlauf nach der Rehabilitation einen positiven Trend auf, der mit einer Reintegrationsquote von 20\% nach einem Jahr allerdings nur sehr schwach ausgeprägt war (Abb. 7). Auch dieser Befund belegt eindeutig, dass in der Praxis der Suchtrehabilitation reintegrative Maßnahmen für Arbeitslose in weitaus stärkerem Maße als bisher erforderlich sind. Das unterstreichen auch die hohen Mittelwerte der ununterbrochenen Arbeitslosigkeit nach der Rehabilitation, die bei allen untersuchten Arbeitslosengruppen dicht an der Grenze hin zur Langzeitarbeitslosigkeit lagen oder diese bereits überschritten hatten.

4. Hingegen war bei den bei Reha-Ende Beschäftigten zu beobachten: Wer nach der Suchtbehandlung erwerbstätig war, hatte gute Chancen, dies für relativ lange Zeit zu bleiben. Das entspricht den Untersuchungsergebnissen von Henkel et al. (2004) [2]. Tab. 1 jedoch macht transparent, dass dies nicht auf alle Gruppen gleichermaßen zutrifft. Zum einen war die Variation in den mittleren Beschäftigungszeiten mit 12 bis 18 Monaten beträchtlich. Zum anderen zeigten die Ergebnisse zum Erwerbsverlauf derjenigen, die als Erwerbstätige in die Suchtbehandlung gekommen waren, dass der Beschäftigtenanteil nach der Rehabilitation kontinuierlich ab- und die Arbeitslosenquote stetig zugenommen hatte (Abb. 8). Das verweist deutlich auf die Notwendigkeit von arbeitsplatzsichernden Maßnahmen für die Erwerbstätigen.

5. In den Ergebnissen der Gruppenvergleiche zur ununterbrochenen Dauer der Arbeitslosigkeit und Beschäftigung sind keine suchtspezifischen Besonderheiten zu erkennen. Sie decken sich weitgehend mit den Befunden, die man aus den allgemeinen Strukturanalysen des Arbeitsmarkts kennt. Prekäre Erwerbsverläufe, d.h. kürzere Beschäftigungs- und längere Arbeitslosigkeitszeiten, sind deutlich häufiger bei gering Qualifizierten zu beobachten (ersatzweise kann hierzu die untere Einkommensgruppe herangezogen werden), bei Frauen, Älteren über 50 Jahren, Ostdeutschen und bei Erwerbspersonen mit gesundheitlichen Einschränkungen (hier: mit langer $\mathrm{Ar}$ beitsunfähigkeit) $[8,14]$. Daher muss die Konzeption von Maßnahmen zur Förderung der Teilhabe am Arbeitsleben, wie in der allgemeinen Arbeitsmarktpolitik auch, vor allem auf diese Gruppen ausgerichtet sein.

Allerdings waren die Suchtrehabilitanden im Vergleich zur Gesamtheit der Arbeitslosen wesentlich länger arbeitslos. Während der Durchschnittswert der Arbeitslosigkeitsdauer in Deutschland in den Jahren 1999 bis 2003 bei rund 8 Monaten in West- und 10 Monaten in Ostdeutschland lag [8], betrug er bei den arbeitslosen Suchtrehabilitanden des Reha-Jahrgangs 2001 allein im 2-JahresZeitraum nach der Rehabilitation 11,9 bzw. 13,6 Monate (Tab.1). Bei Einbeziehung der Behandlungszeit kämen jeweils noch knapp 3 Monate hinzu. Darüber hinaus zeigen andere repräsentative Daten, dass jeder Zweite der bei Behandlungsbeginn Arbeitslosen be- reits vor der Reha-Maßnahme mindestens 12 Monate ununterbrochen ohne Arbeit war, rund 30\% sogar länger als 2 Jahre [9].

Da mit zunehmender Dauer der Arbeitslosigkeit die Reintegrationschancen geringer werden [17], was auch Tab. 1 zeigt (s. Arbeitslosigkeit unter und über 6 Monaten), ist davon auszugehen, dass für einen Teil der Suchtrehabilitanden hohe Risiken bestehen, in schwer umkehrbare Prozesse beruflicher Ausgrenzung zu geraten. Daher sind die arbeitsfördernden Maßnahmen besonders für die Langzeitarbeitslosen zu verbessern und zu intensivieren.

Abschließend sei angemerkt, dass die rehabilitationsstatistischen Daten der BfA, so auch die RSD, keine Informationen über die Suchtproblematik der Rehabilitanden im Zeitraum nach der Behandlung enthalten. Daher lässt sich die Frage, welchen Einfluss die Alkoholabstinenz bzw. -rückfälligkeit auf den Erwerbsverlauf ausübte, nicht unmittelbar empirisch, sondern lediglich im Verbund mit anderen Untersuchungen grob beantworten. Legt man bei den Arbeitslosen, wie in der Forschung nachgewiesen, eine Abstinenzquote von $50 \%$ sechs und zwölf Monate nach der Behandlung zugrunde [1 - 2], dann ist vor dem Hintergrund des lediglich $20 \%$ betragenden Beschäftigtenanteils unter den vor der Behandlung Arbeitslosen (Abb. 7) offensichtlich, dass die Alkoholabstinenz die beruflichen Reintegrationschancen nur in begrenztem Maß verbessert haben konnte. Auch das unterstreicht, dass eine Wiedereingliederung in Arbeit häufig nur erreicht werden kann, wenn die Behandlung der Sucht durch arbeitsfördernde Maßnahmen ergänzt wird. Dass deren Effektivität wiederum nur wesentlich erhöht werden kann, wenn es gelingt, die Massenarbeitslosigkeit durch eine aktive Arbeitsmarkt- und Beschäftigungspolitik substanziell zu verringern, versteht sich von selbst.

\section{Literatur}

${ }^{1}$ Henkel D, Zemlin U, Dornbusch P. Analyse rückfallbeeinflussender Bedingungen bei arbeitslosen Alkoholabhängigen (ARA-Projekt). Teil II: Ergebnisse des Therapieverlaufs und der 6-Monatskatamnese. Sucht aktuell 2004; 11 (1): 21 - 32

${ }^{2}$ Henkel D, Zemlin U, Dornbusch P. Analyse rückfallbeeinflussender Bedingungen bei arbeitslosen Alkoholabhängigen (ARA-Projekt). Teil III: Abstinenz und Rückfall in der 12-Monatskatamnese, Veränderungen im Katamneseverlauf, Unterschiede zwischen Rückfälligen und Abstinenten in der Aufnahme-, Entlass- und Katamnesediagnostik sowie zwischen Erst- und Wiederbehandelten. Sucht aktuell 2004; 11 (2): $5-15$

${ }^{3}$ Zobel M, Missel P, Bachmeier M et al. Effektivität der stationären Suchtrehabilitation - FVS-Katamnese des Entlassjahrgangs 2002 von Fachkliniken für Alkohol- und Medikamentenabhängige. Sucht aktuell 2005; 12 (1): $11-20$

${ }^{4}$ Hümmelink R, Grünbeck P. Sozialmedizinische Prognose nach stationärer Sucht-Rehabilitation. Aktuelle Auswertungen von Routinedaten der BfA. Sucht aktuell 2002; 10 (2): 26-29

${ }^{5}$ Verband Deutscher Rentenversicherungsträger (VDR). VDR-Statistik Rehabilitation, Frankfurt/Main, Jahrgänge 1975 - 2003

${ }^{6}$ Klosterhuis H. Analysemöglichkeiten mit Routinedaten der Sozialversicherung. In: Bengel J, Koch U (Hrsg). Grundlagen der Rehabilitationswissenshaften - Themen, Strategien und Methoden der Rehabilitationsforschung. Berlin, Heidelberg, New York: Springer Verlag, 2000: $451-466$

${ }^{7}$ Bundesministerium für Arbeit und Sozialordnung. Statistisches Taschenbuch. Arbeits- und Sozialstatistik. Berlin, 2004

${ }^{8}$ Bundesagentur für Arbeit. Arbeitsmarktstatistik Nürnberg, Jahrgänge $1995-2003$ 
${ }^{9}$ Henkel D, Zemlin U, Dornbusch P. Analyse rückfallbeeinflussender Bedingungen bei arbeitslosen Alkoholabhängigen (ARA-Projekt). Teil I: Einführung in die Thematik, Projektziele, Untersuchungsanlage und Ergebnisse zu Beginn der Suchttherapie. Sucht aktuell 2003; 10 (2): 5-14

${ }^{10}$ Henkel D. Arbeitslosigkeit, Alkoholkonsum und Alkoholabhängigkeit: nationale und internationale Forschungsergebnisse. In: Henkel D (Hrsg). Sucht und Armut. Alkohol, Tabak, illegale Drogen. Opladen: Leske \& Budrich, 1998: 101 - 136

${ }^{11}$ Henkel D. Arbeitslosigkeit, Alkoholkonsum und Alkoholabhängigkeit: Forschungsergebnisse, Forschungsdefizite und Hypothesen. Abhängigkeiten 1998; 4 (3): 9-29

12 Hollederer A. Arbeitslosigkeit und Gesundheit. Ein Überblick über empirische Befunde und die Arbeitslosen- und Krankenkassenstatistik. Mitteilungen aus der Arbeitsmarkt- und Berufsforschung 2002; 35 (3): $411-428$
${ }^{13}$ Grobe T, Schwartz F. Arbeitslosigkeit und Gesundheit. Gesundheitsberichterstattung des Bundes Heft 13. Berlin: Robert-Koch-Institut, 2003

${ }^{14}$ Almendinger J, Eichhorst W, Walwei U. IAB Handbuch Arbeitsmarkt. Analysen, Daten, Fakten. Frankfurt/Main: Campus, 2005

${ }^{15}$ Schnur P, Zika G. Nur zögerliche Besserung am deutschen Arbeitsmarkt. Projektion des Arbeitskräftebedarfs bis 2020. IAB-Kurzbericht Nr. 12. 2.7.2005

${ }^{16}$ Statistisches Bundesamt. Strukturdaten auf dem Arbeitsmarkt. Wiesbaden, 2004

${ }^{17}$ Mitteilungen aus der Arbeitsmarkt- und Berufsforschung. Nürnberg: Institut für Arbeitsmarkt- und Berufsforschung IAB, 1999; Heft 2

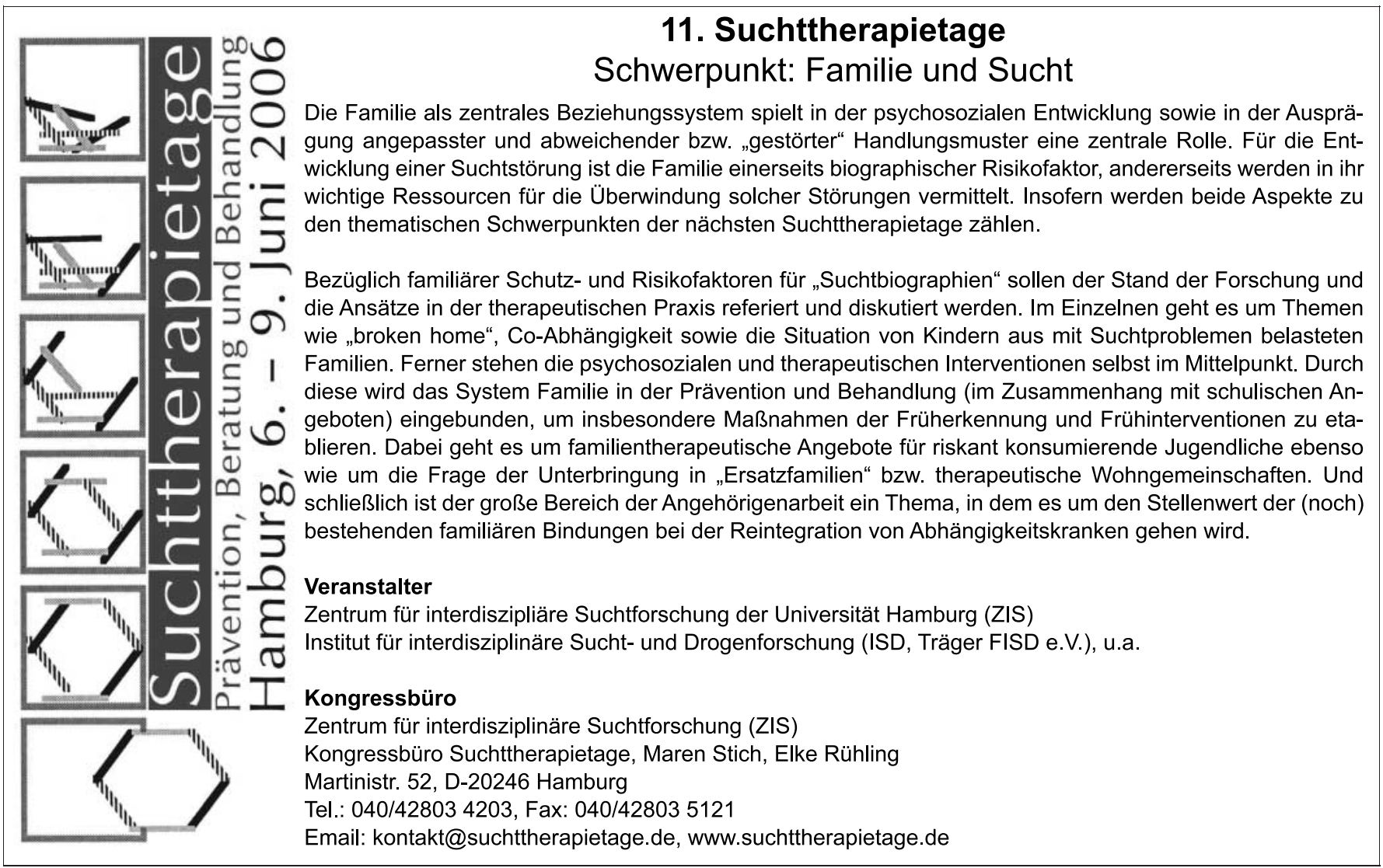

